\title{
Is Repetition of the Contralateral Mammogram of Patients Referred for Unilateral Findings from the Dutch Breast Cancer Screening Necessary?*
}

\author{
Christian Johannes Guillaume Castro ${ }^{1,2}$, Robert Jan Schipper ${ }^{1,2 \#}$, Lori Maria van Roozendaal ${ }^{1,2}$, \\ Mireille van Goethem ${ }^{3}$, Marc Bernardus Irene Lobbes ${ }^{2}$, Marjolein Louisa Smidt ${ }^{1}$ \\ ${ }^{1}$ Department of Surgery, Maastricht University Medical Center, Maastricht, The Netherlands \\ ${ }^{2}$ Department of Radiology, Maastricht University Medical Center, Maastricht, The Netherlands \\ ${ }^{3}$ Department of Radiology, Antwerp University Hospital, Antwerp, Belgium \\ Email: castro.cjg@gmail.com, ${ }^{\#}$ robertjan.schipper@mumc.nl, lori.van.roozendaal@mumc.nl, \\ mireille_van_goethem@hotmail.com,marc.lobbes@mumc.nl,m.smidt@mumc.nl
}

Received August 13, 2013; revised August 28, 2013; accepted September 3, 2013

Copyright (C) 2013 Christian Johannes Guillaume Castro et al. This is an open access article distributed under the Creative Commons Attribution License, which permits unrestricted use, distribution, and reproduction in any medium, provided the original work is properly cited.

\begin{abstract}
Objective: To determine the benefit of contralateral mammogram repetition in patients referred from the Dutch breast cancer screening program for unilateral findings. Material and Methods: 395 patients were referred from screening to our institution for unilateral findings between October 2009 and August 2011. In all patients a bilateral mammogram was repeated and analyzed by experienced breast radiologists. Anonymised data concerning the date of registration of the screening mammogram, the referred side (left/right or bilateral), age, screening's BI-RADS classification, breast density, biopsy results and follow-up were collected. Results: Of the 395 patients referred for a suspicious unilateral finding, a malignancy on the referred breast was confirmed in 144 patients. In addition, a malignancy in the contralateral breast was diagnosed in six patients $(1.5 \%)$. One of these six contralateral malignancies was occult on screening mammogram, but depicted on institutional mammogram only. The remaining five cases were detected in a blinded retrospective analysis by an experienced radiologist and were considered overlooked by the screening radiologists. During follow-up (mean 27.8 months), no contralateral malignancies were detected. Conclusion: Repetition of the two-view mammogram of the contralateral side in patients referred with a unilateral suspicious finding seems unnecessary.
\end{abstract}

Keywords: Breast Neoplasms; Breast Cancer Screening; Conventional Mammography

\section{Introduction}

The Dutch screening program includes women from the age of 50 until 75 . Screening mammograms are performed biannually in mobile units and minimally consist of bilateral two-view mammography (mediolateral oblique and craniocaudal views) [1]. These are then viewed by two independent radiologists to assess the need for referral to a dedicated breast cancer clinic. In case of discrepancies, a third (senior screening) radiologist makes the final decision.

When women are referred to a dedicated breast cancer clinic, Dutch guidelines still recommend repetition of the bilateral two-view mammogram, even when patients are

\footnotetext{
*The authors declare that they have no conflict of interest.

${ }^{\#}$ Corresponding author.
}

referred for only one breast. Arguments for this approach are that images viewed on DVD or CDROM are not of diagnostic quality, but increase in sensitivity when abnormalities are imaged more often (for example, to rule out glandular tissue superposition), or in quality control of the hospital's own hardware. These arguments, however, are debatable and not based on convincing scientific evidence. In contrast, repetition of the exam has several drawbacks: unnecessary patient discomfort [2], unnecessary radiation exposure, and increase in health care costs. Despite arguments in favor of repetition of the complete mammographic exam, repetition of the non-referred side does not seem to have any logical benefits, since the screening is digitalized in 2009. A study of Nederend et al. [3] showed that this improvement of quality led to a significant increased referral rate and cancer detection 
rate of the screening program. Therefore in this study, the aim was to evaluate the added value of repetition of the mammographic exam for patients referred from the Dutch breast cancer screening program for a unilateral suspicious lesion.

\section{Materials and Methods}

In the Netherlands, research covered by the Medical Research Involving Human Subjects Act must be submitted to an accredited medical ethics committee for approval. However, the Act does not cover retrospective research using (coded) data from patient's medical record or patient images. Therefore, our medical ethics committee concluded that the research proposal of the current study does not, under Dutch law, require medical ethical approval (decision number: METC 11-4-137).

All digital (screening) mammograms were performed on a full-field digital mammography system (Selenia, Hologic Inc, Danbury, USA). The mammograms were performed according to a standard protocol, which includes a mediolateral oblique and craniocaudal view of each breast (special views are optional). The mean glandular dose (MGD) for the bilateral two-view mammogram exposed to the patient was $5.8 \mathrm{mGy}$ (Standard deviation (SD) $1 \mathrm{mGy}$ ) (Information from the southern breast cancer screening region of the Netherlands, Eindhoven, Netherlands). With use of the breast tissue weighting factor 0.12 , according to the International Commission on Radiological Protection (ICRP) 103 report, this MGD corresponds to an average effective radiation dose of $(5.8 \mathrm{mGy} \times 0.12$ breast tissue weighting factor) $0.7 \mathrm{mSv}$ [4]. All screening mammograms were viewed by two independent experienced screening radiologists. In case of discrepancies, a third (senior) screening radiologists makes the final decision on referral or not.

The radiologists classified the findings according to an adapted version of the American College of Radiology Breast Imaging Reporting and Data System (BI-RADS) [5]. In the Dutch screening setting, findings can only be scored as being BI-RADS 0,4 or 5 . If the mammogram shows an abnormality, women are referred to a breast clinic of their choice.

We retrospectively collected all the data from women referred to our dedicated breast cancer clinic in a study period from October 2009 to August 2011. When referred, the bilateral diagnostic two-view mammogram was repeated for all patients on a full-field digital mammography system (Senopgraphe Essential, GE Healthcare, Waukesha, WI, USA), with a mean MGD for a bilateral two-view mammogram of $6 \mathrm{mGy}$ (SD $1.1 \mathrm{mGy}$ ), which correlates with an average effective radiation dose of $0.72 \mathrm{mSv}$. Images of both breasts were reviewed and compared with screening mammograms by experienced breast radiologists.

In our work-up for these women, tissue superposition is suggested by performing the repeated mammography, a minimum of one additional special view of the area of interest, and a negative targeted ultrasound. Tissue superposition is confirmed as final diagnosis after six months of follow-up and an unaltered mammographic exam. If a cyst is suggested as cause for the referred abnormality, it is aspirated. A control mammogram should confirm the disappearance of the culprit abnormality. In all solid lesions (either benign or malignant), final diagnosis is made by image-guided large core biopsy. In general, a preoperative breast MRI is performed in breast cancer cases to assess tumour extent and evaluate the contralateral breast.

The anonymized data of all patients concerning the date of registration of the screening mammogram, the referred side (left/right or bilateral), age, screening BI-RADS, breast density, final diagnosis, biopsy results and breast MRI results was gathered in a database. Descriptive statistics were performed using the SPSS statistical software package, version 18.0 (SPSS, Chicago, IL, USA).

\subsection{Retrospective Analysis of Contralateral Malignancies}

To investigate whether newly detected contralateral lesions were (not) mammographically occult on screening mammography, all mammograms of the patients with contralateral breast cancer were retrospectively reviewed by an independent experienced radiologist (over 24 years of experience in screening and clinical mammography), who was blinded for every study results and aim. A validation set of 14 random mammograms from patients referred for confirmed unilateral breast cancer were added to this data set for blinding purposes. If a contralateral lesion was detected, the radiologist scored the mammogram using the regular BI-RADS classification [5]. If no contralateral lesion was depicted, the radiologist received the information of the preoperative breast MRI. With this information the radiologist retrospectively scored the lesion as being "occult", a "minimal sign" or an "obvious finding" on both the screening mammogram and our institution's mammogram.

\subsection{Follow-Up}

We registered the follow-up results of the enrolled patients and checked out the follow-up results for possible missed breastcancer in the contralateral breast. In the Netherlands during the 1-year after breast cancer diagnosis, follow-up consists of a physical examination every three months. In the second year the physical examina- 
tion takes place every 6 months and in the third year only once a year. Every year also a bilateral two-view mammogram is performed.

\section{Results}

In our screening region, 31,901 women were invited for breast cancer screening from October 2009 to August 2011. A total of 28,112 women (88\%) responded and of these, a total of 402 patients were referred to our dedicated breast cancer clinic. Seven patients were referred for bilateral suspicious lesions and were thus excluded from this study, leaving 395 patients referred for a unilateral suspicious lesion (Figure 1), with a mean age of 59 years (standard deviation (SD) 7.6 years).

Mean time between screening and institutional mammogram was 14.3 days (SD 3.3 days). Of the 395 patients referred for a suspicious unilateral lesion, no malignancy was detected in 245 patients $(62 \%)$. In the re- maining 150 patients, a unilateral malignancy was detected in 144 patients. In the remaining six patients an additional cancer was diagnosed in the contralateral breast (Figure 1). Table 1 provided additional information on which exams these cancers were visible and includes final histopathological diagnosis and size (case numbers are designated 1 to 6 ).

In case 1 to 5 , bilateral breast cancer was detected. In case 6 , no malignancy was detected on the referred side, but the cancer was detected on the contralateral side. Final diagnosis of these contralateral cases showed four invasive ductal carcinoma (IDC) with a mean diameter of $1.2 \mathrm{~cm}$ (SD $0.35 \mathrm{~cm}$ ), and two ductal carcinoma in situ, with a mean diameter of $2.15 \mathrm{~cm}$ (SD $0.15 \mathrm{~cm})$.

When re-evaluated by our institutional radiologists after referral, three contralateral malignancies were directly detected with the aid of the screening and institutional mammogram (cases 2 to 4 ). Case 2 consisted of a spicular mass in the contralateral breast, detected on both screen-

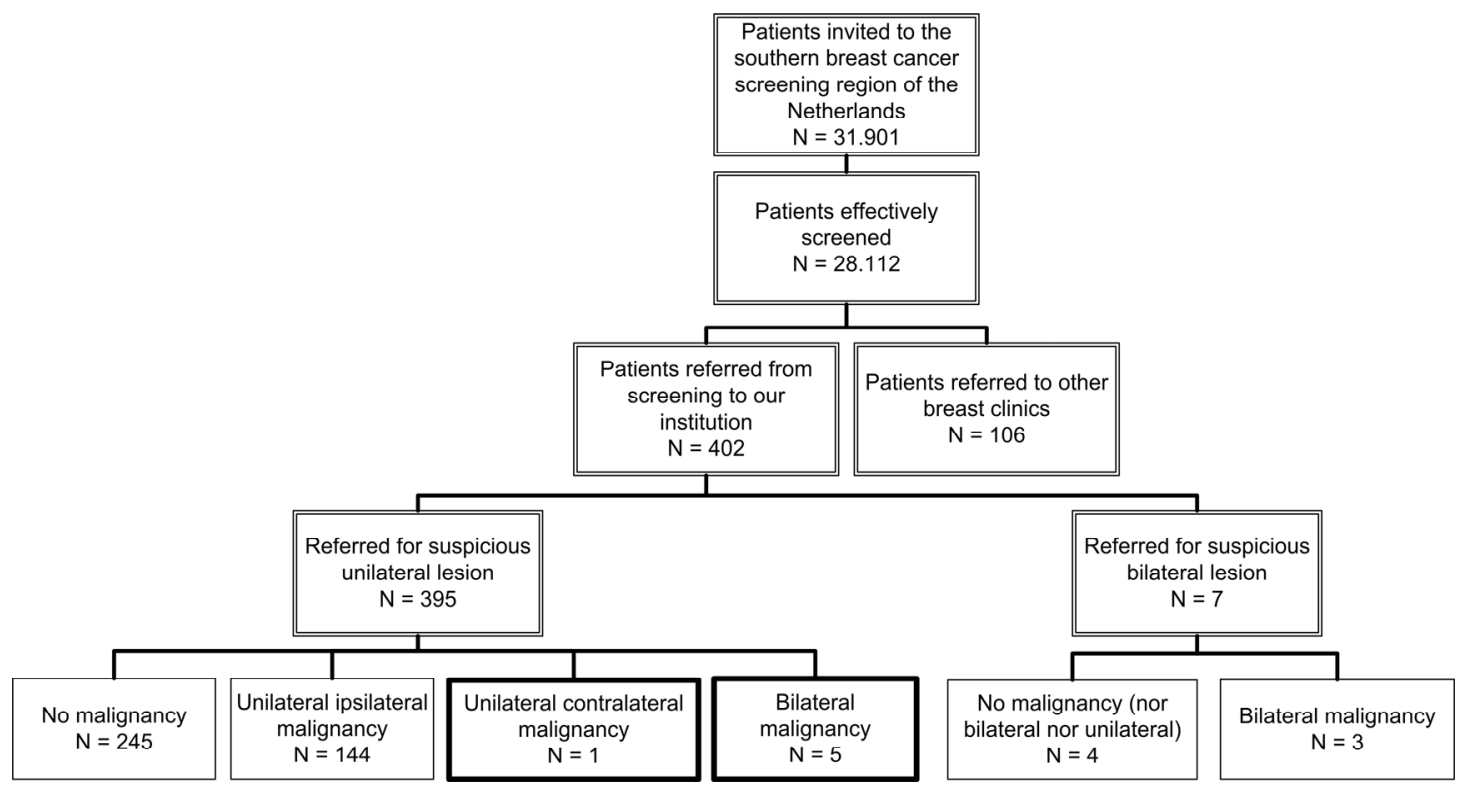

Figure 1. Women screened during a study period of 2 years and referred to our institution for diagnostic analysis.

Table 1. Findings of the screening mammogram, institutional mammogram and the breast MRI with the pathology results of the cases with a contralateral malignancy.

\begin{tabular}{|c|c|c|c|c|c|c|c|c|}
\hline \multirow[t]{2}{*}{ Case } & \multicolumn{2}{|c|}{ Screening MG } & \multicolumn{2}{|c|}{ Institutional MG } & \multicolumn{2}{|c|}{ Breast MRI } & \multicolumn{2}{|c|}{ Pathology } \\
\hline & $\begin{array}{c}\text { Referred } \\
\text { breast }\end{array}$ & $\begin{array}{c}\text { Contralateral } \\
\text { breast }\end{array}$ & $\begin{array}{c}\text { Referred } \\
\text { breast }\end{array}$ & $\begin{array}{c}\text { Contralateral } \\
\text { breast }\end{array}$ & $\begin{array}{c}\text { Referred } \\
\text { breast }\end{array}$ & $\begin{array}{c}\text { Contralateral } \\
\text { breast }\end{array}$ & $\begin{array}{c}\text { Referred } \\
\text { breast }\end{array}$ & $\begin{array}{c}\text { Contralateral } \\
\text { breast }\end{array}$ \\
\hline 1 & + & - & + & - & + & + & IDC (Ø $1.5 \mathrm{~cm})$ & IDC (Ø $1.4 \mathrm{~cm})$ \\
\hline 2 & + & - & + & + & + & + & IDC (Ø $1.1 \mathrm{~cm})$ & IDC (Ø $1.6 \mathrm{~cm})$ \\
\hline 3 & + & - & + & + & + & + & IDC (Ø $1.9 \mathrm{~cm})$ & DCIS (Ø $2.3 \mathrm{~cm})$ \\
\hline 4 & + & - & + & + & + & + & IDC (Ø $1.8 \mathrm{~cm})$ & IDC (Ø $1.0 \mathrm{~cm})$ \\
\hline 5 & + & - & - & - & + & + & IDC (Ø $0.9 \mathrm{~cm})$ & IDC (Ø $0.7 \mathrm{~cm})$ \\
\hline 6 & + & - & + & - & - & + & No malignancy & DCIS (Ø $2.0 \mathrm{~cm})$ \\
\hline
\end{tabular}

+: Suspect lesion, -: No suspect lesion, MG: mammogram, IDC: Invasive ductal carcinoma, DCIS: Ductal carcinoma in situ. 
ing and institutional mammogram. Case 4 showed an irregular mass in the contralateral breast, detected on both screening and institutional mammogram. In case 3 , our radiologist detected fine linear microcalcifications in the contralateral breast on the institutional mammogram, not on the screening mammogram. In case 5, no lesion was detected in both breasts on the institutional mammogram. A breast MRI was performed because of a discrepancy between the screening and institutional mammogram, which showed a bilateral breast cancer. Interestingly, all cancers were correctly identified with help of breast MRI, both ipsilateral and contralateral cancers (Table 1).

Figure 2 shows an example of a contralateral (minimal sign) lesion (case 1), overlooked with both the screening and institutional mammogram, but detected with breast MRI.

\subsection{Retrospective Analysis of the Contralateral Cancers}

In order to determine whether these contralateral malignancies were occult or simply overlooked, we performed a retrospective analysis of these cases by an independent and highly experienced breast radiologist, who was blinded for every study results and aim. For further blinding purposes, we imbedded the cases in a validation set of mammograms. This dataset consisted of 14 random cases with unilateral breast cancer. In this set, the radiologist correctly detected five out of six contralateral malignancies on the screening mammogram which we therefore considered to be overlooked by the screening radiologists. Figure 3 shows an example of fine linear microcalcifications (case 3), which were overlooked by the screening radiologist, but detected on the institutional mammogram. In retrospect, knowing that there are microcalcifications, these microcalcifications were seen on the screening mammogram. As described earlier in only one case (case 6), fine linear microcalcifications were detected on the institutional mammogram, which were occult on the screening mammogram. Table 2 provided additional information and an overview of the BI-RADS of the detected contralateral malignancies.

\subsection{Follow-Up}

The follow-up of the 144 patients with a unilateral malignancy showed in 139 patients no contralateral malignancies. Two patients were lost to follow-up and three patients are deceased. The follow-up range was $12-40$ months with a mean time of 27.8 months (SD 7.3 months). Every patient had at least a 1-year control mammogram.

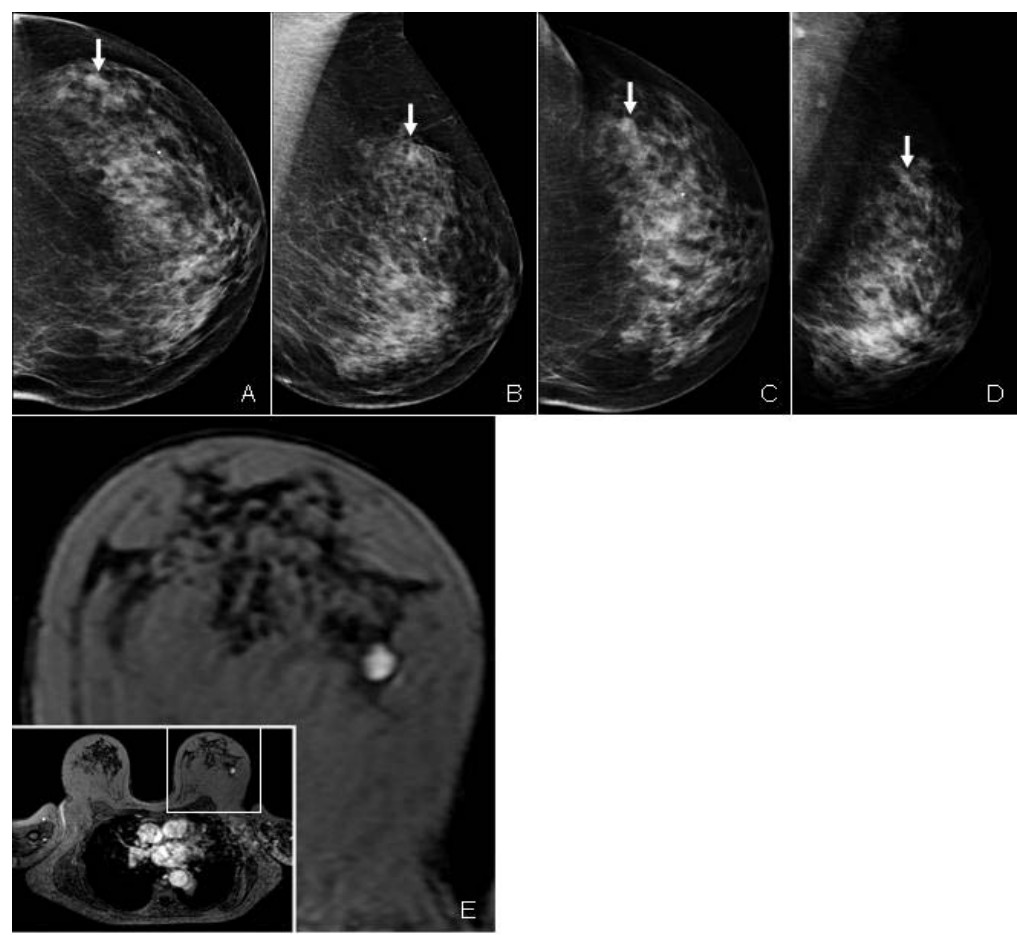

Figure 2. Contralateral (minimal sign) lesion in the left upper outer quadrant of the contralateral breast, overlooked with mammography but detected using breast MRI. (A) Craniocaudal and (B) mediolateral oblique screening mammogram of the contralateral breast, showing an oval, ill-defined mass (see arrows). Repetition of the mammography (C), (D) shows a similar presentation of the lesion (see arrow). (E) Dynamic contrast enhanced breast MRI showing a slightly lobulated, well-defined, homogeneously enhancing mass in the lateral part of the left breast with a diameter of $1.5 \mathrm{~cm}$. 


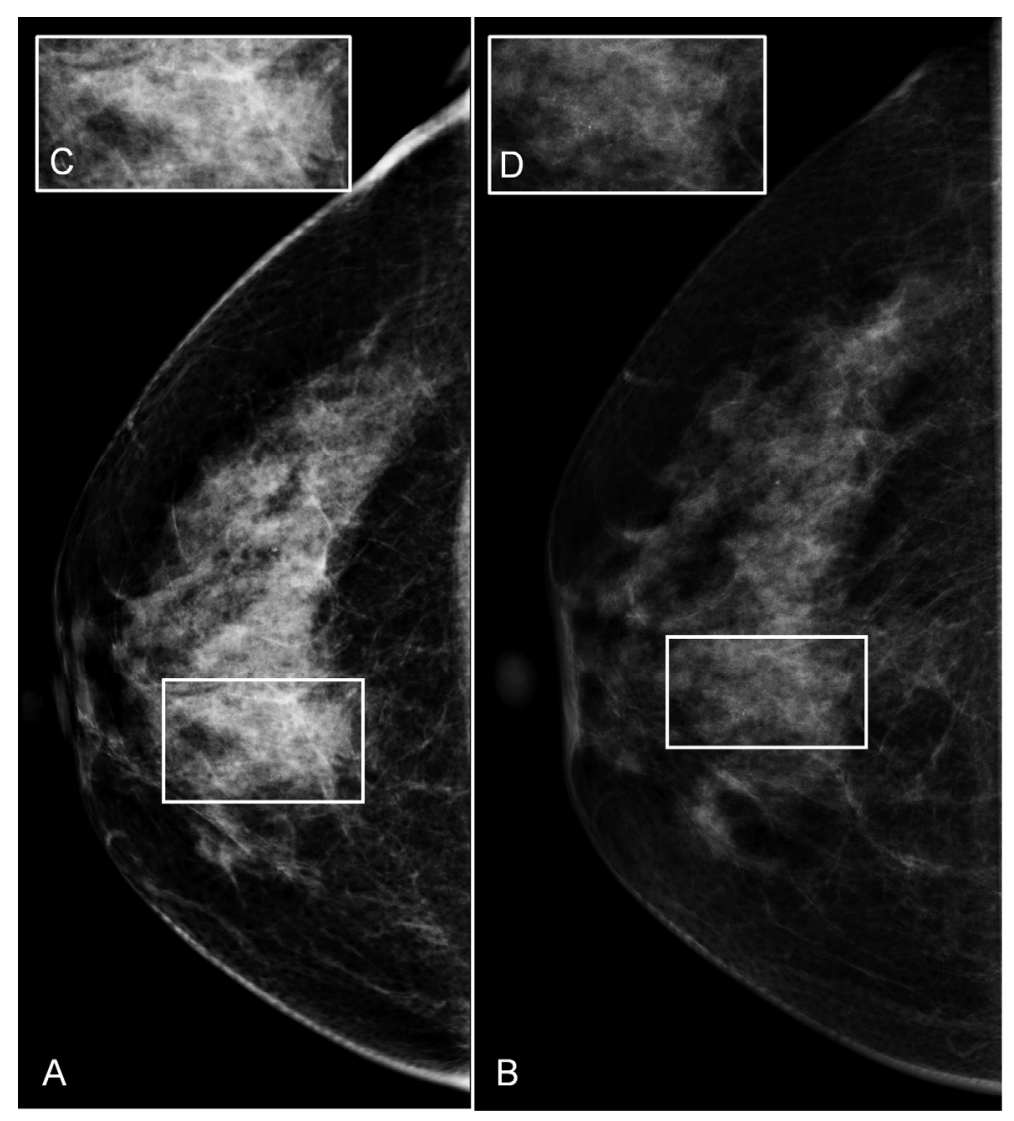

Figure 3. Contralateral (minimal sign) microcalcifications in the right lower inner quadrant of the contralateral breast. (A) Craniocaudal screening mammogram of the contralateral breast, showing singular microcalcifications, overlooked by the screening radiologist. (C) Increased area with the microcalcifications, which were seen during the retrospective analysis. Repetition of the craniocaudal mammography (B), (D) shows obvious microcalcifications (obvious finding). In this setting was the repetition of the contralateral breast an added value, but also without the repetition an experienced radiologist would have taken a biopsy to definitive exclude DCIS.

\section{Discussion}

In the era of analogue mammography, repetition of the two-view mammogram of both sides was preferred because of the potential quality difference between screening mammograms and institutional mammograms. According to Bluekens et al. the performance of digital mammography in the detection of DCIS and invasive ductal carcinoma is significantly better than analogue mammography [6]. The digital mammography for screening was introduced over a period of several years (region per region) and as a result, many hospitals performed digital mammography while their screening region still used analogue techniques. Nowadays, the complete screening program is digitalized, but Dutch guidelines have not adapted accordingly.

In these guidelines, women referred from the breast cancer screening program traditionally undergo repetition of the complete mammographic examination, even if referred for a unilateral finding [7]. Arguments for this approach are that images viewed on DVD or CDROM are not of diagnostic quality, increase in sensitivity when abnormalities are imaged more often (for example, to rule out glandular tissue superposition), or quality control of the hospital's own hardware. These arguments, however, are debatable and not based on convincing scientific evidence. It can be argued that repetition of the mammogram confirms the patient's identity in a highthroughput setting like the screening program. Repetition of the mammogram of the referred side confirms the identity of the patient and the culprit lesion, and it is the authors' opinion that it can sometimes aid in analysis of superposition shadows of fibroglandular tissue. Despite these arguments in favor of repetition of the complete mammographic exam, repetition of the non-referred side does not seem to have any logical benefits, which we can conclude out of our results. This study showed that out of 395 patients referred for a unilateral suspicious finding since the introduction of digital screening mammography in our region and a study period of 2 years, only six patients were diagnosed with a contralateral cancer. Five out of six contralateral malignancies were retrospectively visible on screening mammograms and were considered 
Table 2. Results (lesion: occult/minimal sign/obvious finding) of the women with a contralateral malignancy retrospectively.

\begin{tabular}{ccccc}
\hline Case & & Retrospective (Screening) & Retrospective (Institutional MG) \\
\hline & & Contralateral breast & Contralateral breast \\
\hline 1 & + & BI-RADS 4a (minimal sign) & + & BI-RADS 4b (minimal sign) \\
2 & + & BI-RADS 4c (obvious finding) & + & BI-RADS 4c (obvious finding) \\
3 & + & BI-RADS 4a (minimal sign) & + & BI-RADS 4c (obvious finding) \\
4 & + & BI-RADS 4a (minimal sign) & + & BI-RADS 4c (obvious finding) \\
5 & + & BI-RADS 4b (minimal sign) & + & BI-RADS 4c (obvious finding) \\
6 & - & BI-RADS 1 (occult) & + & BI-RADS 4a (minimal sign) \\
\hline
\end{tabular}

+: contralateral lesion depicted, -: no contralateral lesion depicted.

overlooked by the screening radiologists. Only a single malignant lesion proved to be occult on the screening mammogram was visible as fine linear microcalcifications on our institutional mammogram. Also during the follow-up no contralateral malignancy was detected, allowing us to rule out missed cancers. Therefore, our study did not demonstrate any convincing benefit for repeating the contralateral mammogram when women are referred from the screening program for a unilateral finding.

Different studies have shown that synchronous bilateral breast cancer (SBBC) is relatively rare. According to Kheirelseid et al. [8], about $2.4 \%$ of all referred patients are diagnosed with bilateral breast cancer. Other studies report an incidence rate varying from $1 \%$ to $3 \%$ $[8,9]$. These findings are in line with our results, showing that approximately $0.75 \%$ (3 SBBC/402 referred patients) of all referred patients were diagnosed with bilateral breast cancer. About 1.3\% (5 additional contralateral lesions/395 patients referred for a unilateral suspicious lesion) of all patients referred for a suspicion of a unilateral lesion were finally diagnosed with a bilateral breast cancer.

In this study, the majorities of the contralateral malignancies were correctly identified by the blinded retrospective analysis of our experienced breast radiologist and were considered overlooked. Only in one referral an abnormality was detected which was truly occult on the screening mammography, but was detected by the institutional mammography and breast MRI.

The most likely explanation why the contralateral lesions were overlooked at the screening was the phenomenon of the "satisfaction of search", also commonly known as the "happy eye syndrome", or "instant happiness syndrome". The observation of a suspicious lesion may mislead a screening radiologist into not looking carefully for another (contralateral) lesion [10]. This study showed that almost all contralateral lesions could be depicted retrospectively, albeit that they were "minimal signs". A study of Ashman et al. [10] showed that most of the contralateral breast cancers in patients who had been referred for a unilateral lesion had been missed at screening.

Omission of the (unnecessary) contralateral mammogram might have several benefits: a reduction of health care costs could be achieved, reduction of radiation exposure and as a result, also radiation-induced cancer, and less patient discomfort. In the Netherlands about 1.1 million women get an invitation for the screening mammogram. About $900.000(80 \%)$ of the women participate at the screening program annually [1]. These numbers correlate with the numbers of our screening region, where 31.901 women were invited and 28.112 women participated $(88 \%)$. The overall costs of the breast cancer screening program are about 44 million euro [11]. Two out of 100 patients are referred for further diagnostic analysis, i.e. 18.000 patients yearly [1].

The cost of a bilateral two-view mammogram in our institution is 45 euro. In theory, this means if half of this fee could be saved for omitting the contralateral mammogram of every referred patient, an estimated total of 450.000 euro (18.000 referred patients $\times 25$ euro) could be saved.

With respect to dose reduction, the average glandular dose of a mammogram is very low, but the acquisition of unnecessary images is against the radiologists' ALARA principe (As Low As Reasonably Achievable). For example, the ICRP 103 report (International Commission on Radiological Protection) quantifies radiation risk by using the concept of effective dose [4]. As described earlier the MGD of a bilateral and a unilateral two-view mammogram in our institution was 6 and $3 \mathrm{mGy}$ respectively, which correlates with an average effective dose of 0.72 and $0.36 \mathrm{mSv}$ respectively. These numbers agree with the study of Hendrick [4]. The United States National Academy of Sciences Biologic Effects of Ionizing Radiation VII Group [12], give a Lifetime Attributable Risk of mortality due to breast cancer of 0.73 cases per 100.000 women per $1 \mathrm{mGy}$ exposure of the breast. This means if the contralateral two-view mammogram could be omitted ( $3 \mathrm{mGy}), 0.4$ radiation-induced breast cancer cases could be spared yearly $((18.000$ patients $\times(0.73$ 
radiation-induced breast cancers/100.000 women per mGy)) $\times 3$ mGy exposure) .

Also, unnecessary patient discomfort could be avoided. A study of Keemers-Gels et al. [2] showed that more than $70 \%$ of the women experienced pain and discomfort scored as mild to severe during mammography. Omission of the contralateral mammogram would thus lead to less pain and discomfort, and would aid in the motivation of patients to participate in screening programs.

This study has some limitations. Firstly, the study had a limited study population, resulting into only six cases of overlooked contralateral malignancies. However, since digitalization of screening mammography in our region was introduced in October 2009, it was not possible to include more patients in this time frame. Secondly, the validation set of 14 patients added for blinding to the data set of six contralateral breast malignancies was limited and consisted of solely abnormal mammograms. Nonetheless, we think this was a good method to evaluate whether lesions were overlooked by screening radiologists, since our independent radiologist was highly experienced in viewing mammograms and was unaware of the study aims and results at the time of analysis. Thirdly, the study focuses on the results of the Dutch screening program and is therefore mainly interesting for the Dutch setting.

However, when extrapolating the result of this study to screening programs in other countries, one should consider that the referral rate in the Netherlands has traditionally been low in comparison to other breast cancer screening programs in Europe and in the USA. On the contrary, the Netherlands has a comparable breast cancer detection rate and one of the highest breast cancer mortality reduction [13]. So, the proportion of cases with a (screening occult) contra lateral tumour in our cohort is probably higher when comparing to other countries.

We think that in the digital era, repetition of exams from referred patients, especially of the contralateral breast in patients referred for a suspicious unilateral finding, should not be performed as standard procedure. It is recommended that these exams are re-evaluated on dedicated viewing stations and that quality of the images should be assessed first. Based on this review, specific repeated views and additional imaging of a breast could then be considered.

\section{Conclusion}

Repetition of the two-view mammogram of the contralateral side in patients referred with a unilateral suspicious finding is unnecessary, since only one additional in situ carcinoma was detected on institutional mammogram out of 395 patients. The five other contralateral cancers that were found in our study population were overlooked by the screening radiologists. We would recommend re-assessing the digital screening mammogram first and decide on additional imaging or interventions based on these exams.

\section{Acknowledgements}

This study was supported by the Carla Boetes Fund.

\section{Ethical Standards}

For this study, ethics approval and informed consent for the use of the (coded) images was waived according to Dutch law (decision number: METC 11-4-137).

\section{REFERENCES}

[1] G. A. Miltenburg, P. H. Peeters, J. Fracheboud and H. J. Collette, "Seventeen-Year Evaluation of Breast Cancer Screening: The DOM Project, The Netherlands. Diagnostisch Onderzoek (Investigation) Mammacarcinoom," British Journal of Cancer, Vol. 78, No. 7, 1998, pp. 962965.

[2] M. E. Keemers-Gels, R. P. Groenendijk, J. H. van den Heuvel, C. Boetes, P. G. Peer and T. H. Wobbes, "Pain Experienced by Women Attending Breast Cancer Screening," Breast Cancer Research and Treatment, Vol. 60, No. 3, 2000, pp. 235-240. http://dx.doi.org/10.1023/A:1006457520996

[3] J. Nederend, L. E. Duijm, M. W. Louwman, J. H. Groenewoud, A. B. Donkers-van Rossum and A. C. Voogd, "Impact of Transition from Analog Screening Mammography to Digital Screening Mammography on Screening Outcome in The Netherlands: A Population-Based Study," Annals of Oncology, Vol. 23, No. 12, 2012, pp. 30983103. http://dx.doi.org/10.1093/annonc/mds 146

[4] R. E. Hendrick, "Radiation Doses and Cancer Risks from Breast Imaging Studies," Radiology, Vol. 257, No. 1, 2010, pp. 246-253.

http://dx.doi.org/10.1148/radiol.10100570

[5] D. Vanel, "The American College of Radiology (ACR) Breast Imaging and Reporting Data System (BI-RADS): A Step towards a Universal Radiological Language?" European Journal of Radiology, Vol. 61, No. 2, 2007, p. 183. http://dx.doi.org/10.1016/i.ejrad.2006.08.030

[6] A. M. Bluekens, R. Holland, N. Karssemeijer, M. J. Broeders and G. J. den Heeten, "Comparison of Digital Screening Mammography and Screen-Film Mammography in the Early Detection of Clinically Relevant Cancers: A Multicenter Study," Radiology, Vol. 265, No. 3, 2012, pp. 707-714. http://dx.doi.org/10.1148/radiol.12111461

[7] NABON, "Guideline Mamma Carcinoma 2012," NABON, The Netherlands, 2012.

http://www.oncoline.nl/mammacarcinoom

[8] E. A. Kheirelseid, H. Jumustafa, N. Miller, C. Curran, K. Sweeney, C. Malone, et al., "Bilateral Breast Cancer: Analysis of Incidence, Outcome, Survival and Disease Characteristics," Breast Cancer Research and Treatment, Vol. 126, No. 1, 2011, pp. 131-140.

[9] D. E. Heron, L. T. Komarnicky, T. Hyslop, G. F. Sch- 
wartz and C. M. Mansfield, "Bilateral Breast Carcinoma: Risk Factors and Outcomes for Patients with Synchronous and Metachronous Disease," Cancer, Vol. 88, No. 12, 2000, pp. 2739-2750.

http://dx.doi.org/10.1002/1097-0142(20000615)88:12<27 39::AID-CNCR12>3.0.CO;2-J

[10] C. J. Ashman, J. S. Yu and D. Wolfman, "Satisfaction of Search in Osteoradiology," American Journal of Roentgenology, Vol. 175, No. 2, 2000, pp. 541-544. http://dx.doi.org/10.2214/ajr.175.2.1750541

[11] J. H. Groenewoud, J. D. Otten, J. Fracheboud, G. Draisma, B. M. van Ineveld, R. Holland, et al., "Cost-Effectiveness of Different Reading and Referral Strategies in Mammography Screening in the Netherlands," Breast Cancer
Research and Treatment, Vol. 102, No. 2, 2007, pp. 211218.

[12] Academies NRCotN, "Health Risk from Exposure to Low Levels of Ionizing Radiation: BEIR VII, Phase 2Committee to Assess Health Risks from Exposure to Low Levels of Ionizing Radiation," National Academies Press, Washington DC, 2006.

[13] J. G. Elmore, C. Y. Nakano, T. D. Koepsell, L. M. Desnick, C. J. D'Orsi and D. F. Ransohoff, "International Variation in Screening Mammography Interpretations in Community-Based Programs," Journal of National Cancer Institute, Vol. 95, No. 18, 2003, pp. 1384-1393. http://dx.doi.org/10.1093/jnci/djg048

\section{List of Abbreviations}

ALARA: As Low As Reasonably Achievable

DCIS: Ductal carcinoma in situ

EUSOBI: European society of breast imaging

ICRP: International Commission on Radiological Protection

IDC: Invasive ductal carcinoma

MG: Mammogram

MGD: Mean glandular dose

mGy: Milligray

MRI: Magnetic Resonance Imaging

mSv: Millisievert

SBBC: Synchronous bilateral breast cancer

SD: Standard deviation 